\title{
AMPLIACÃO DOS CRITÉRIOS DE DIVISIBILIDADE PARA OUTROS VALORES
}

\section{EXTENSION OF CRITERIA IN DIVISIBILITY FOR OTHERS VALUES}

Ana Paula Souza Santos, Rianne dos Santos Garcia, Antonio Carlos Tamarozzi

Universidade Federal de Mato Grosso do Sul, Grupo PET Matemática, Três Lagoas, MS.

E-mail: anasantosj5_@hotmail.com

RESUMO - Este artigo é resultado de um trabalho de pesquisa desenvolvido pelo grupo PETMAT - Programa de Educação Tutorial da UFMS/CPTL, cujo objetivo é demonstrar os critérios de divisibilidade usuais, normalmente encontrados nos livros didáticos brasileiros, a partir do ensino fundamental e apresentar critérios para outros números primos. As demonstrações dos resultados podem ser construídas a partir de congruências e um lema sobre divisibilidade de um produto $a . b$, onde $a$ e $b$ são números inteiros positivos, primos entre si. Além do interesse do tema para a iniciação cientifica dos discentes do grupo PETMAT, o projeto objetiva disponibilizar material educativo para aplicação nas escolas.

Palavras-chave: Inteiros; Primos; Divisibilidade; Critérios; Demonstração.

ABSTRACT - This article is the result of a research project carried out by PETMAT - Tutorial Education Program group of UFMS/CPTL, whose aim is to demonstrate the usual divisibility criteria, usually found in Brazilian textbooks from primary and present criteria for another Prime numbers. The results demonstrations can be built from congruence and a lemma about divisibility of a a.b product, where a and $b$ are positive integers, relatively primes. Besides the issue of interest to the scientific initiative of students of PETMAT group, the project aims to provide educational materials for use in schools.

Keywords: Integer; Prime; Divisibility; Criteria; Demonstration. 


\section{INTRODUÇÃO}

Critérios de divisibilidade consistem em algoritmos algébricos simples para verificar se um dado número inteiro é divisível por outro número inteiro. Tais critérios normalmente são introduzidos aos alunos no inicio do ensino fundamental e tem um papel importante no ensino de números inteiros, pois despertam a atenção do aluno ao permitirem resoluções rápidas de problemas que envolvem divisibilidades e fatorações.

Os critérios de divisibilidade mais comuns encontrados nos livros didáticos, por exemplo (ANDRINI et al, 2012) e (IEZZI et al, 2009), restringem-se a testes de divisões com os números inteiros do intervalo de 2 a 11 e contemplam aplicações de sua utilização, sem apresentarem as demonstrações da validade dos resultados. Este trabalho de pesquisa desenvolvido pelo grupo PETMAT Programa de Educação Tutorial, vinculado ao curso de Matemática da UFMS/CPTL, objetiva estender os critérios de divisibilidade para outros números primos conjuntamente com a demonstração dos resultados. Além do interesse do tema para a iniciação cientifica dos discentes do grupo PETMAT, o projeto objetiva disponibilizar material educativo para aplicação nas escolas. Os exemplos de alguns critérios de divisibilidade não usuais foram sugeridos pelo material educativo disponibilizado

em <pessoal.sercomtel.com.br/matematica/fund am/naturais/divisibilidade.htm>, acesso em 15 jun. 2016.

\section{METODOLOGIA}

O trabalho foi desenvolvido inicialmente com a pesquisa bibliográfica do tema para a verificação de como o mesmo é desenvolvido no ensino fundamental e médio. Enquanto que, a pesquisa bibliográfica (DOMINGUES; IEZZI, 2003) evidencia as técnicas que podem ser empregadas nas demonstrações.

As seguintes definições são básicas para o processo de demonstrações neste trabalho.

Definição: Dados dois inteiros $a \mathrm{e} b$, com $a \neq 0$, dizemos que a divide $b$ (denotamos $a \mid b$ ) se existe $c$ inteiro tal que $b$ $=\mathrm{ac}$.

Definição: Sejam a, b, n inteiros com n $>0$. Dizemos que a é congruente $a b(\bmod n)$ $($ denota-se $a \equiv b(\bmod n))$ se $n \mid(b-a)$.

Destacamos os seguintes resultados que utilizaremos ao longo do trabalho

Proposição: Se $a \equiv b(\bmod n)$ e $c \equiv d$ $(\bmod n)$ então $(a+c) \equiv(b+d)(\bmod n)$ e $a c \equiv$ bd $(\bmod n)$.

Demonstração: $n|(b-a), n|(d-c)$ $\Rightarrow \mathrm{n} \mid(\mathrm{b}+\mathrm{d})-(\mathrm{a}+\mathrm{c}) \Rightarrow(\mathrm{a}+\mathrm{c}) \equiv$

$(b+d)($ módulo $n)$, e bd $-a c=b(d-c)$ $+((b-a) \Rightarrow n \mid(b d-a c) \Rightarrow b d \equiv a c(\bmod n)$. 
Proposição: Dados os inteiros positivos a e $n \geq 1$, existem únicos inteiros $0 \leq$ ai $\leq 9$ de tal forma que $a=a_{n} 10^{n}+\cdots$ $+a_{2} 10^{2}+a_{1} 10+a_{0}\left(^{*}\right)$.

A proposição anterior mostra que todo inteiro positivo pode ser decomposto de forma única na base 10 .

\section{RESULTADOS}

Utilizando congruências e a decomposição na base 10, podemos demonstrar alguns dos critérios de divisibilidade usuais apresentados no ensino fundamental.

Critério de divisibilidade por 2: Um número natural a é divisível por 2 quando ele é par.

Este resultado segue facilmente da decomposição de a na base 10. De fato, suponhamos que a tenha a decomposição $\left(^{*}\right)$ acima. Como cada potencia de 10 é divisível por 2 , temos $10^{\mathrm{k}} \equiv 0$ para todo $\mathrm{k} \geq$ 1, logo pela propriedade de congruências de somas, segue que $a \equiv a_{0}(\bmod 2)$ ou seja a é par se, e somente se, o ultimo algarismo for par.

Critério de divisibilidade por 5: Um número natural a é divisível por 5 quando terminar em 0 ou 5.

Novamente usando a decomposição na base $10(*)$ acima e tendo em vista que $10^{\mathrm{k}} \equiv 0(\bmod 5)$ para todo $\mathrm{k} \geq 1$, resulta que $\mathrm{a} \equiv \mathrm{a}_{0}(\bmod 5)$, ou seja a divisibildade por 5 depende do algarismo da unidade ser divisível por 5 . Como 0 ou 5 são estes únicos algarismos, segue o resultado.

Critério de Divisibilidade por 3 e 9: Um número é divisível por 3 quando a soma dos seus algarismos for divisível por 3.

Observemos que $10 \equiv 1(\bmod 3)$, logo pela propriedade de congruência temos que $10^{\mathrm{k}} \equiv 1(\bmod 3)$ para todo $\mathrm{k} \geq 1$. Assim, usando a decomposição na base $10\left(^{*}\right)$ acima, vemos que $a \equiv\left(a_{0}+a_{1}+a_{2}+\ldots+a_{n}\right)(\bmod$ 3), de onde segue o resultado. A divisibilidade por 9 é tratada de forma análoga, pois $10^{\mathrm{k}} \equiv 1(\bmod 9)$ para todo $\mathrm{k} \geq$ 1.

Critério de divisibilidade por 7: Dado um número $\mathrm{a}=\mathrm{a}_{\mathrm{n}} 10^{\mathrm{n}}+\cdots+\mathrm{a}_{2} 10^{2}+$ $a_{1} 10+a_{0}$ tem-se que a é divisível por 7 se, e somente se, $b=\left(a_{n} 10^{n-1}+\cdots+a_{2} 10+\right.$ $\left.a_{1}\right)-2 a_{0}$ for divisível por 7 .

Observemos que se $\mathrm{b}$ for divisível por 7 então a é divisível por 7 .

Hipótese: $\left(a_{n} 10^{n-1}+\cdots+a_{2} 10+a_{1}\right)-2 a_{0}=$ $7 k$, onde $k \in Z$

Tese: $\mathrm{a}=7 \mathrm{k}^{\prime}$, onde $\mathrm{k}^{\prime} \in \mathrm{Z}$.

Temos, $\quad \mathrm{a}=\mathrm{a}_{\mathrm{n}} 10^{\mathrm{n}}+\cdots+\mathrm{a}_{2} 10^{2}+$ $a_{1} 10+a_{0}=10\left(a_{n} 10^{n-1}+\cdots+a_{2} 10+a_{1}\right)+$ $\mathrm{a}_{0}$, como pela hipótese temos que, $\mathrm{a}_{\mathrm{n}} 10^{\mathrm{n}-1}+\cdots+\mathrm{a}_{2} 10+\mathrm{a}_{1}=7 \mathrm{k}+2 \mathrm{a}_{0} \quad \mathrm{e}$ portanto $\mathrm{a}=10\left(7 \mathrm{k}+2 \mathrm{a}_{0}\right)+\mathrm{a}_{0}=70 \mathrm{k}+$ $20 \mathrm{a}_{0}+\mathrm{a}_{0}+70 \mathrm{k}+21 \mathrm{a}_{0}=7\left(10 \mathrm{k}+3 \mathrm{a}_{0}\right)=$ $7 \mathrm{k}^{\prime}$, onde $\mathrm{k}^{\prime}=10 \mathrm{k}+3 \mathrm{a}_{0}, \mathrm{k}^{\prime} \in \mathrm{Z}$. 
Logo temos que 7|a. Portanto a proposição está demonstrada.

$(\Rightarrow)$ Se a for divisível por 7 então b é divisível por 7 .

Hipótese: $\mathrm{a}_{\mathrm{n}} 10^{\mathrm{n}}+\cdots+\mathrm{a}_{2} 10^{2}+\mathrm{a}_{1} 10+\mathrm{a}_{0}=$ $7 \mathrm{k}^{\prime}$, onde $\mathrm{k}^{\prime} \in \mathrm{Z}$

Tese: $\mathrm{b}=7 \mathrm{t}, \mathrm{t} \in \mathrm{Z}$

Temos que $a=7 k^{\prime}$, dessa forma temos $\mathrm{a}_{\mathrm{n}} 10^{\mathrm{n}}+\cdots+\mathrm{a}_{2} 10^{2}+\mathrm{a}_{1} 10+\mathrm{a}_{0}=$ $7 \mathrm{k}^{\prime} \Leftrightarrow 10\left(\mathrm{a}_{\mathrm{n}} 10^{\mathrm{n}-1}+\cdots+\mathrm{a}_{2} 10+\mathrm{a}_{1}\right)+$ $\mathrm{a}_{0}=7 \mathrm{k}^{\prime}$, somamos então $-21 \mathrm{a}_{0}$ de ambos os lados da igualdade temos, $10\left(a_{n} 10^{n-1}+\right.$. $\left.\cdot+\mathrm{a}_{2} 10+\mathrm{a}_{1}\right)+\mathrm{a}_{0}-21 \mathrm{a}_{\sigma}=7 \mathrm{k}^{\prime}-21 \mathrm{a}_{0}$ $\Leftrightarrow 10\left(\mathrm{a}_{\mathrm{n}} 10^{\mathrm{n}-1}+\cdots+\mathrm{a}_{2} 10+\mathrm{a}_{1}\right)-$ $20 \mathrm{a}_{0}=7 \mathrm{k}^{\prime}-21 \mathrm{a}_{0} \Leftrightarrow \quad 10\left(\mathrm{a}_{\mathrm{n}} 10^{\mathrm{n}-1}+\cdots\right.$ $\left.+\mathrm{a}_{2} 10+\mathrm{a}_{1}-2 \mathrm{a}_{0}\right)=7\left(\mathrm{k}^{\prime}-3 \mathrm{a}_{0}\right)$ $\Leftrightarrow 10\left(\mathrm{a}_{\mathrm{n}} 10^{\mathrm{n}-1}+\cdots+\mathrm{a}_{2} 10+\mathrm{a}_{1}-2 \mathrm{a}_{0}\right)=$ $7 \mathrm{t}, \mathrm{t}=\mathrm{k}^{\prime}-3 \mathrm{a}_{0}, \mathrm{t} \in \mathrm{Z}$. Mais ainda, pelo lema "Se p é um número primo e $\mathrm{p} \mid \mathrm{ab}$ então $p \mid a$ ou $p \mid b^{\prime \prime}$ temos que7| $\left(a_{n} 10^{n-1}+\cdots\right.$ $\left.+\mathrm{a}_{2} 10+\mathrm{a}_{1}-2 \mathrm{a}_{0}\right)$, uma vez que 7 não é um divisor de 10. Logo temos que 7|b e a proposição está demonstrada.

\section{Critério de divisibilidade por 13}

O número $\quad \mathrm{a}=\mathrm{a}_{\mathrm{n}} 10^{\mathrm{n}-1}+\cdots$ $+\mathrm{a}_{2} 10^{2}+\mathrm{a}_{1} 10+\mathrm{a}_{0}$ é divisível por $13 \mathrm{se}$, e somete se, $b=\left(a_{n} 10^{n-1}+\cdots+a_{2} 10+\right.$ $\left.a_{1}\right)+4 a_{0}$ for divisível por 13 .

Demonstração:

$(\Leftarrow)$ Se b é divisível por 13 então a é divisível por 13
Hipótese: $\quad\left(a_{n} 10^{n-1}+\cdots+a_{2} 10+a_{1}\right)+$ $4 \mathrm{a}_{0}=13 \mathrm{k}$, onde $\mathrm{k} \in \mathrm{Z}$

Tese: $\mathrm{a}=13 \mathrm{k}^{\prime}$, onde $\mathrm{k}^{\prime} \in \mathrm{Z}$

Temos, $\quad \mathrm{a}=\mathrm{a}_{\mathrm{n}} 10^{\mathrm{n}}+\cdots+\mathrm{a}_{2} 10^{2}+$ $a_{1} 10+a_{0}=10\left(a_{n} 10^{n-1}+\cdots+a_{2} 10+a_{1}\right)+$ $\mathrm{a}_{0}$, como acima pela hipótese temos que, $a_{n} 10^{n-1}+\cdots+a_{2} 10+a_{1}=13 k-4 a_{0} \quad e$ portanto $\mathrm{a}=10\left(13 \mathrm{k}+4 \mathrm{a}_{0}\right)+\mathrm{a}_{0}=130 \mathrm{k}-$ $40 \mathrm{a}_{0}+\mathrm{a}_{0}=130 \mathrm{k}-39 \mathrm{a}_{0}=13(10 \mathrm{k}-$ $\left.3 \mathrm{a}_{0}\right)=13 \mathrm{k}^{\prime}$, onde $\mathrm{k}^{\prime}=10 \mathrm{k}-3 \mathrm{a}_{0} \mathrm{k}^{\prime} \in \mathrm{Z}$ Logo temos que 13|a. Portanto a proposição está demonstrada.

$(\Rightarrow)$ Se a for divisível por 13 então b é divisível por 13

Hipótese: $\quad \mathrm{a}_{\mathrm{n}} 10^{\mathrm{n}}+\cdots+\mathrm{a}_{2} 10^{2}+$ $\mathrm{a}_{1} 10+\mathrm{a}_{0}=13 \mathrm{k}^{\prime}$, onde $\mathrm{k}^{\prime} \in \mathrm{Z}$

$$
\text { Tese: }\left(\mathrm{a}_{\mathrm{n}} 10^{\mathrm{n}-1}+\cdots+\mathrm{a}_{2} 10+\mathrm{a}_{1}\right)+
$$

$4 \mathrm{a}_{0}=13 \mathrm{t}, \mathrm{t} \in \mathrm{Z}$

Temos que $a=13 \mathrm{k}^{\prime}$, dessa forma temos $\mathrm{a}_{\mathrm{n}} 10^{\mathrm{n}}+\cdots+\mathrm{a}_{2} 10^{2}+\mathrm{a}_{1} 10+\mathrm{a}_{0}=$ $13 \mathrm{k}^{\prime} \Leftrightarrow 10\left(\mathrm{a}_{\mathrm{n}} 10^{\mathrm{n}-1}+\cdots+\mathrm{a}_{2} 10+\mathrm{a}_{1}\right)+$ $\mathrm{a}_{0}=13 \mathrm{k}^{\prime}$, somamos então $-39 \mathrm{a}_{0}$ de ambos os lados da equação temos, $10\left(\mathrm{a}_{\mathrm{n}} 10^{\mathrm{n}-1}+\cdots\right.$ $\left.+\mathrm{a}_{2} 10+\mathrm{a}_{1}\right)+\mathrm{a}_{0}-39 \mathrm{a}_{0}=7 \mathrm{k}^{\prime}-39 \mathrm{a}_{0}$ $\Leftrightarrow 10\left(\mathrm{a}_{\mathrm{n}} 10^{\mathrm{n}-1}+\cdots+\mathrm{a}_{2} 10+\mathrm{a}_{1}\right)-$ $40 \mathrm{a}_{0}=7 \mathrm{k}^{\prime}-39 \mathrm{a}_{0} \Leftrightarrow \quad 10\left(\mathrm{a}_{\mathrm{n}} 10^{\mathrm{n}-1}+\cdots\right.$ $\left.+\mathrm{a}_{2} 10+\mathrm{a}_{1}-4 \mathrm{a}_{0}\right)=13\left(\mathrm{k}^{\prime}-3 \mathrm{a}_{0}\right)$ $\Leftrightarrow 10\left(\mathrm{a}_{\mathrm{n}} 10^{\mathrm{n}-1}+\cdots+\mathrm{a}_{2} 10+\mathrm{a}_{1}-4 \mathrm{a}_{0}\right)=$ $13 \mathrm{t}, \mathrm{t}=\mathrm{k}^{\prime}-3 \mathrm{a}_{0}, \mathrm{t} \in \mathrm{Z}$. Mais ainda pelo lema "Se p é um número primo e $p \mid a b$ então $p \mid a$ ou $p \mid b^{\prime \prime}$ temos que como $13 \mid 10$ então $13 \mid\left(a_{n} 10^{n-1}+\cdots+a_{2} 10+a_{1}-4 a_{0}\right)$ 
Logo temos que 13|b. Portanto a proposição está demonstrada.

\section{Critério de divisibilidade por 17}

$\mathrm{O}$ número $\mathrm{a}=\mathrm{a}_{\mathrm{n}} 10^{\mathrm{n}}+\cdots+\mathrm{a}_{2} 10^{2}+$ $\mathrm{a}_{1} 10+\mathrm{a}_{0}$ é divisível por 17 se, e somente se, $b=\left(a_{n} 10^{n-1}+\cdots+a_{2} 10+a_{1}\right)-5 a_{0}$ for divisível por 17.

Demonstração:

$(\Leftarrow)$ Se b for divisível por 17 então a é divisível por 17.

Hipótese: $\quad\left(\mathrm{a}_{\mathrm{n}} 10^{\mathrm{n}-1}+\cdots+\mathrm{a}_{2} 10+\mathrm{a}_{1}\right)-$ $5 \mathrm{a}_{0}=17 \mathrm{k}$, onde $\mathrm{k} \in \mathrm{Z}$

Tese: $\mathrm{a}=7 \mathrm{k}^{\prime}$, onde $\mathrm{k}^{\prime} \in \mathrm{Z}$

Temos, $\quad \mathrm{a}=\mathrm{a}_{\mathrm{n}} 10^{\mathrm{n}}+\cdots+\mathrm{a}_{2} 10^{2}+$ $\mathrm{a}_{1} 10+\mathrm{a}_{0}=10\left(\mathrm{a}_{\mathrm{n}} 10^{\mathrm{n}-1}+\cdots+\mathrm{a}_{2} 10+\mathrm{a}_{1}\right)+$ $\mathrm{a}_{0}$, como acima pela hipótese temos que, $\mathrm{a}_{\mathrm{n}} 10^{\mathrm{n}-1}+\cdots+\mathrm{a}_{2} 10+\mathrm{a}_{1}=7 \mathrm{k}+2 \mathrm{a}_{0} \quad$ e portanto $\mathrm{a}=10\left(7 \mathrm{k}+2 \mathrm{a}_{0}\right)+\mathrm{a}_{0}=70 \mathrm{k}+$ $20 \mathrm{a}_{0}+\mathrm{a}_{0}+70 \mathrm{k}+21 \mathrm{a}_{0}=7\left(10 \mathrm{k}+3 \mathrm{a}_{0}\right)=$ $7 \mathrm{k}^{\prime}$, onde $\mathrm{k}^{\prime}=10 \mathrm{k}+3 \mathrm{a}_{0} \quad \mathrm{k}^{\prime} \in \mathrm{Z}$.

Logo temos que $7 \mid a$. Portanto a proposição está demonstrada.

$(\Rightarrow)$ Se a for divisível por 7 então b é divisível por 7.

Hipótese: $\mathrm{a}_{\mathrm{n}} 10^{\mathrm{n}}+\cdots+\mathrm{a}_{2} 10^{2}+\mathrm{a}_{1} 10+\mathrm{a}_{0}=$ $7 \mathrm{k}^{\prime}$, onde $\mathrm{k}^{\prime} \in \mathrm{Z}$

Tese: $\mathrm{b}=7 \mathrm{t}, \mathrm{t} \in \mathrm{Z}$

Temos que $a=7 k^{\prime}$, dessa forma temos $\mathrm{a}_{\mathrm{n}} 10^{\mathrm{n}}+\cdots+\mathrm{a}_{2} 10^{2}+\mathrm{a}_{1} 10+\mathrm{a}_{0}=$ $7 \mathrm{k}^{\prime} \Leftrightarrow 10\left(\mathrm{a}_{\mathrm{n}} 10^{\mathrm{n}-1}+\cdots+\mathrm{a}_{2} 10+\mathrm{a}_{1}\right)+$ $\mathrm{a}_{0}=7 \mathrm{k}^{\prime}$, somamos então $-21 \mathrm{a}_{0}$ de ambos os lados da equação temos, $10\left(\mathrm{a}_{\mathrm{n}} 10^{\mathrm{n}-1}+\cdots\right.$ $\left.+\mathrm{a}_{2} 10+\mathrm{a}_{1}\right)+\mathrm{a}_{0}-21 \mathrm{a}_{0}=7 \mathrm{k}^{\prime}-21 \mathrm{a}_{0}$ $\Leftrightarrow 10\left(\mathrm{a}_{\mathrm{n}} 10^{\mathrm{n}-1}+\cdots+\mathrm{a}_{2} 10+\mathrm{a}_{1}\right)-$ $20 \mathrm{a}_{0}=7 \mathrm{k}^{\prime}-21 \mathrm{a}_{0} \Leftrightarrow \quad 10\left(\mathrm{a}_{\mathrm{n}} 10^{\mathrm{n}-1}+\cdots\right.$ $\left.+\mathrm{a}_{2} 10+\mathrm{a}_{1}-2 \mathrm{a}_{0}\right)=7\left(\mathrm{k}^{\prime}-3 \mathrm{a}_{0}\right)$ $\Leftrightarrow 10\left(\mathrm{a}_{\mathrm{n}} 10^{\mathrm{n}-1}+\cdots+\mathrm{a}_{2} 10+\mathrm{a}_{1}-2 \mathrm{a}_{0}\right)=$ $7 t, t=k^{\prime}-3 a_{0}, t \in Z$. Mais ainda pelo lema "Se $\mathrm{p}$ é um número primo e $\mathrm{p} \mid \mathrm{ab}$ então $\mathrm{p} \mid \mathrm{a}$ ou $p \mid b^{\prime \prime}$ temos que como $7 \mid 10$ então $7 \mid\left(a_{n} 10^{n-1}+\cdots+a_{2} 10+a_{1}-2 a_{0}\right)$

Logo temos que $7 \mid b$. Portanto a proposição está demonstrada.

\section{Critério de divisibilidade por 29}

Seja um número $\mathrm{a}=\mathrm{a}_{\mathrm{n}} 10^{\mathrm{n}}+\cdots$ $+\mathrm{a}_{2} 10^{2}+\mathrm{a}_{1} 10+\mathrm{a}_{0}$ é divisível por 29 se, e somente se, $b=\left(a_{n} 10^{n-1}+\cdots+a_{2} 10+\right.$ $\left.a_{1}\right)+3 a_{0}$ for divisível por 29 .

Demonstração:

$(\Leftarrow)$ Se b for divisível por 29 então a é divisível por 29.

Hipótese: $\quad\left(\mathrm{a}_{\mathrm{n}} 10^{\mathrm{n}-1}+\cdots+\mathrm{a}_{2} 10+\mathrm{a}_{1}\right)+$ $3 \mathrm{a}_{0}=29 \mathrm{k}$, onde $\mathrm{k} \in \mathrm{Z}$ Tese: $\mathrm{a}=29 \mathrm{k}^{\prime}$, onde $\mathrm{k}^{\prime} \in \mathrm{Z}$

Temos, $\quad \mathrm{a}=\mathrm{a}_{\mathrm{n}} 10^{\mathrm{n}}+\cdots+\mathrm{a}_{2} 10^{2}+$ $\mathrm{a}_{1} 10+\mathrm{a}_{0}=10\left(\mathrm{a}_{\mathrm{n}} 10^{\mathrm{n}-1}+\cdots+\mathrm{a}_{2} 10+\mathrm{a}_{1}\right)+$ $\mathrm{a}_{0}$, como acima pela hipótese temos que, $\mathrm{a}_{\mathrm{n}} 10^{\mathrm{n}-1}+\cdots+\mathrm{a}_{2} 10+\mathrm{a}_{1}=29 \mathrm{k}-3 \mathrm{a}_{0} \quad \mathrm{e}$ portanto $\mathrm{a}=10\left(29 \mathrm{k}-3 \mathrm{a}_{0}\right)+\mathrm{a}_{0}=290 \mathrm{k}+$ $30 \mathrm{a}_{0}+\mathrm{a}_{0}=290 \mathrm{k}-29 \mathrm{a}_{0}=29\left(\mathrm{k}-\mathrm{a}_{0}\right)=$ $29 \mathrm{k}^{\prime}$, onde $\mathrm{k}^{\prime}=\mathrm{k}-\mathrm{a}_{0} \quad \mathrm{k}^{\prime} \in \mathrm{Z}$. 
Logo temos que 29|a. Portanto a proposição está demonstrada.

$(\Rightarrow)$ Se a for divisível por 29 então b é divisível por 29.

Hipótese: $\quad \mathrm{a}_{\mathrm{n}} 10^{\mathrm{n}}+\cdots+\mathrm{a}_{2} 10^{2}+$ $\mathrm{a}_{1} 10+\mathrm{a}_{0}=29 \mathrm{k}^{\prime}$, onde $\mathrm{k}^{\prime} \in \mathrm{Z}$

Tese: $\mathrm{b}=29 \mathrm{t}, \mathrm{t} \in \mathrm{Z}$

Temos que $a=29 \mathrm{k}^{\prime}$, dessa forma temos $\mathrm{a}_{\mathrm{n}} 10^{\mathrm{n}}+\cdots+\mathrm{a}_{2} 10^{2}+\mathrm{a}_{1} 10+\mathrm{a}_{0}=$ $29 \mathrm{k}^{\prime} \Leftrightarrow 10\left(\mathrm{a}_{\mathrm{n}} 10^{\mathrm{n}-1}+\cdots+\mathrm{a}_{2} 10+\mathrm{a}_{1}\right)+$ $\mathrm{a}_{0}=29 \mathrm{k}^{\prime}$, somamos então $29 \mathrm{a}_{0}$ de ambos os lados da equação temos, $10\left(a_{n} 10^{n-1}+\cdots\right.$ $\left.+\mathrm{a}_{2} 10+\mathrm{a}_{1}\right)+\mathrm{a}_{0}+29 \mathrm{a}_{\vartheta}=29 \mathrm{k}^{\prime}+29 \mathrm{a}_{0}$ $\Leftrightarrow 10\left(\mathrm{a}_{\mathrm{n}} 10^{\mathrm{n}-1}+\cdots+\mathrm{a}_{2} 10+\mathrm{a}_{1}\right)+$ $30 \mathrm{a}_{0}=29 \mathrm{k}^{\prime}+29 \mathrm{a}_{0} \Leftrightarrow \quad 10\left(\mathrm{a}_{\mathrm{n}} 10^{\mathrm{n}-1}+\cdots\right.$ $\left.+\mathrm{a}_{2} 10+\mathrm{a}_{1}-3 \mathrm{a}_{0}\right)=29\left(\mathrm{k}^{\prime}-\mathrm{a}_{0}\right)$ $\Leftrightarrow 10\left(\mathrm{a}_{\mathrm{n}} 10^{\mathrm{n}-1}+\cdots+\mathrm{a}_{2} 10+\mathrm{a}_{1}-3 \mathrm{a}_{0}\right)=$ $29 t, t=k^{\prime}+a_{0}, t \in Z$. Mais ainda pelo lema "Se $\mathrm{p}$ é um número primo e $\mathrm{p} \mid \mathrm{ab}$ então $\mathrm{p} \mid \mathrm{a}$ ou $p \mid b^{\prime \prime}$ temos que como 29|10 então $29 \mid\left(a_{n} 10^{n-1}+\cdots+a_{2} 10+a_{1}-3 a_{0}\right)$

Logo temos que 29|b. Portanto a proposição está demonstrada.

\section{Critério de divisibilidade por 31}

O número $\mathrm{a}=\mathrm{a}_{\mathrm{n}} 10^{\mathrm{n}}+\cdots+\mathrm{a}_{2} 10^{2}+$ $\mathrm{a}_{1} 10+\mathrm{a}_{0}$ é divisível por 31 se, e somente se, $b=\left(a_{n} 10^{n-1}+\cdots+a_{2} 10+a_{1}\right)-3 a_{0}$ for divisível por 31.

Demonstração:

$(\Leftarrow)$ Se b for divisível por 31 então a é divisível por 31.
Hipótese: $\quad\left(\mathrm{a}_{\mathrm{n}} 10^{\mathrm{n}-1}+\cdots+\mathrm{a}_{2} 10+\mathrm{a}_{1}\right)-$ $3 \mathrm{a}_{0}=31 \mathrm{k}$, onde $\mathrm{k} \in \mathrm{Z}$

Tese: $\mathrm{a}=31 \mathrm{k}^{\prime}$, onde $\mathrm{k}^{\prime} \in \mathrm{Z}$

Temos, $\quad \mathrm{a}=\mathrm{a}_{\mathrm{n}} 10^{\mathrm{n}}+\cdots+\mathrm{a}_{2} 10^{2}+$ $a_{1} 10+a_{0}=10\left(a_{n} 10^{n-1}+\cdots+a_{2} 10+a_{1}\right)+$ $\mathrm{a}_{0}$, como acima pela hipótese temos que, $\mathrm{a}_{\mathrm{n}} 10^{\mathrm{n}-1}+\cdots+\mathrm{a}_{2} 10+\mathrm{a}_{1}=31 \mathrm{k}+3 \mathrm{a}_{0} \quad \mathrm{e}$ portanto $\mathrm{a}=10\left(31 \mathrm{k}+3 \mathrm{a}_{0}\right)+\mathrm{a}_{0}=310 \mathrm{k}+$ $30 \mathrm{a}_{0}+\mathrm{a}_{0}=310 \mathrm{k}+31 \mathrm{a}_{0}=31(10 \mathrm{k}+$ $\left.\mathrm{a}_{0}\right)=31 \mathrm{k}^{\prime}$, onde $\mathrm{k}^{\prime}=10+\mathrm{a}_{0}, \mathrm{k}^{\prime} \in \mathrm{Z}$. Logo temos que 31|a. Portanto a proposição está demonstrada.

$(\Rightarrow)$ Se a for divisível por 31 então b é divisível por 31.

Hipótese: $\mathrm{a}_{\mathrm{n}} 10^{\mathrm{n}}+\cdots+\mathrm{a}_{2} 10^{2}+\mathrm{a}_{1} 10+\mathrm{a}_{0}=$ $31 \mathrm{k}^{\prime}$, onde $\mathrm{k}^{\prime} \in \mathrm{Z}$

Tese: $b=31 t, t \in Z$

Temos que $a=7 k^{\prime}$, dessa forma temos $\mathrm{a}_{\mathrm{n}} 10^{\mathrm{n}}+\cdots+\mathrm{a}_{2} 10^{2}+\mathrm{a}_{1} 10+\mathrm{a}_{0}=$ $31 \mathrm{k}^{\prime} \Leftrightarrow 10\left(\mathrm{a}_{\mathrm{n}} 10^{\mathrm{n}-1}+\cdots+\mathrm{a}_{2} 10+\mathrm{a}_{1}\right)+$ $\mathrm{a}_{0}=31 \mathrm{k}^{\prime}$, somamos então $-31 \mathrm{a}_{0}$ de ambos os lados da equação temos, $10\left(a_{n} 10^{n-1}+\cdots\right.$ $\left.+\mathrm{a}_{2} 10+\mathrm{a}_{1}\right)+\mathrm{a}_{0}-31 \mathrm{a}_{0}=31 \mathrm{k}^{\prime}-31 \mathrm{a}_{0}$ $\Leftrightarrow 10\left(\mathrm{a}_{\mathrm{n}} 10^{\mathrm{n}-1}+\cdots+\mathrm{a}_{2} 10+\mathrm{a}_{1}\right)-$ $30 \mathrm{a}_{0}=31\left(\mathrm{k}^{\prime}-\mathrm{a}_{0}\right) \Leftrightarrow \quad 10\left(\mathrm{a}_{\mathrm{n}} 10^{\mathrm{n}-1}+\cdots\right.$ $\left.+\mathrm{a}_{2} 10+\mathrm{a}_{1}-3 \mathrm{a}_{0}\right)=31 \mathrm{t}, \mathrm{t}=\left(\mathrm{k}^{\prime}-\right.$ $a_{0}$ ), $t \in Z$. Mais ainda pelo lema "Se $p$ é um número primo e $p \mid a b$ então $p \mid a$ ou $p \mid b^{\prime \prime}$ temos que como 31|10 então $31 \mid\left(a_{n} 10^{n-1}+\cdots+a_{2} 10+a_{1}-3 a_{0}\right)$

Logo temos que 31|b. Portanto a proposição está demonstrada. 


\section{DISCUSSÃO}

Nosso trabalho identifica duas abordagens diferenciadas para demonstrações dos critérios de divisibilidade. A partir do teste de divisibilidade para o divisor $d \geq 7$, somente a utilização de congruências não é suficiente para a obtenção dos resultados, fazendo-se necessário a utilização de outras técnicas da Teoria dos números.

Observemos que os exemplos de critérios aqui estudados podem ser acessíveis aos alunos do ensino básico, quando realizada uma leitura prática dos mesmos. Por exemplo, o critério de divisibilidade por 29 admite a seguinte adaptação: “Um número é divisível por 29 quando o triplo do último algarismo, subtraído do número que não contém este último algarismo, resultar em um número divisível por 29".

\section{CONCLUSÕES}

A utilização dos critérios de divisibilidade é extremamente útil para a resolução de problemas cotidianos que envolvam divisão e, em ambientes escolares, despertam o interesse dos estudantes com resoluções rápidas, dadas sob a forma de algoritmos. O trabalho mostra que é possível ampliar os testes de divisibilidade para outros valores e que as justificativas dos funcionamentos resultam de técnicas aritméticas simples da Teoria dos Números.
Empregando metodologias similares, podem ser obtidos critérios de divisibilidade para números superiores aos estudados neste trabalho.

\section{REFERÊNCIAS}

ANDRINI, A.; VACONCELLOS, M. J. Praticando matemática: 60 ano. 3. ed. São Paulo: Editora do Brasil, 2012.

CRITÉRIOS DE DIVISIBILIDADE. Disponível em: $<$ www.somatematica.com.br/fundam/critdiv. php>. Acesso em: 20 jun. 2016.

DOMINGUES, H. H.; IEZZI, G. Álgebra moderna. 4. ed. reform. São Paulo: Atual, 2003.

IEZZI, G.; DOLCE, O.; MACHADO, A. Matemática e realidade: 60 ano. 6. ed. São Paulo: Atual, 2009. 